\title{
On the Efficiency of Feasible Solutions of a Multicriteria Assignment Problem
}

\author{
Ahmed Bufardi ${ }^{*}$ \\ Department of Environmental Engineering, Swiss Federal Institute of Aquatic Science and Technology (EAWAG), \\ Switzerland
}

\begin{abstract}
The particular structure of the assignment problem made of it a very popular subject of study and an important research tool in operations research and management science. In addition to the importance that the assignment problem represents in its own, it can appear as a relaxation of more complex combinatorial optimization problems. That is why the assignment problem has received great attention from the operations research community. The assignment problem may appear as an optimization problem with multiple objectives. In this paper, we address the problem of efficiency of feasible solutions of a multicriteria assignment problem. It is done in two steps. In the first step, we determine whether or not a given feasible solution of a multicriteria assignment problem is efficient. In a second step, if the feasible solution is not efficient, we provide an efficient solution that dominates it. The proposed method consists of transforming the original problem into an assignment problem with side constraints for which solution techniques already exist.
\end{abstract}

\section{INTRODUCTION}

The (standard) assignment problem consists of assigning a number of tasks to an equal number of agents (each agent is assigned to exactly one task and each task has exactly one agent assigned to perform it) in such a way to minimize the overall cost of assigning agents to tasks, given the cost of the assignment of each agent to each task. The mathematical formulation of the standard assignment problem (SAP) is as follows:

$$
\begin{aligned}
& \min Z(X)=\sum_{i=1}^{n} \sum_{j=1}^{n} c_{i j} X_{i j} \\
& \text { subject to } \sum_{j=1}^{n} X_{i j}=1, i=1, \ldots, n \\
& \sum_{i=1}^{n} X_{i j}=1, j=1, \ldots, n \\
& X_{i j} \in\{0,1\}, i, j=1, \ldots, n
\end{aligned}
$$

where for all $i, j=1, \ldots, n, c_{i j}$ is the cost of assigning agent $i$ to task $\mathrm{j}, \mathrm{X}_{\mathrm{ij}}=1$ means that agent $\mathrm{i}$ is assigned to task $\mathrm{j}$ and $\mathrm{X}_{\mathrm{ij}}=0$ means that agent $\mathrm{i}$ is not assigned to task $\mathrm{j}$.

The first set of constraints implies that each agent is assigned to one and only one task and the second set of constraints implies that to each task is assigned one and only one agent.

It is worth to mention that the constraints $X_{\mathrm{ij}}=0$ or $\mathrm{X}_{\mathrm{ij}}=$ 1 for all $i, j=1, \ldots, n$ can be replaced by the constraints $X_{i j} \geq$ 0 for all $i, j=1, \ldots, n$ because the assignment problem satisfies the important property of total unimodularity.

In addition to the minimization of assignment cost, an assignment problem may consider other objective functions

*Address correspondence to this author at the Department of Environmental Engineering, Swiss Federal Institute of Aquatic Science and Technology (EAWAG), Switzerland; E-mail: ahmed.bufardi@eawag.ch such as the minimization of completion time. When the assignment problem is considered with the minimization of assignment cost as the objective function, it is called the cost minimizing assignment problem.

It is worth to recall that the assignment problem has been used in a variety of application contexts such as personnel scheduling, manpower planning and resource allocation.

Sonia and Puri [1] reported that various methodologies including primal-dual algorithms, simplex-like methods, cost operation algorithms, forest algorithms and relaxation techniques have been proposed in the literature to solve the cost minimizing assignment problem.

The well-known Hungarian method developed by Kuhn and published in 1955 [2] is recognized to be the first practical method for solving the SAP [3]. Due to the important impact of the seminal paper [2] on the research related to the assignment problem, the Naval Research Logistics journal that published [2] decided to reprint it in 2005 [4] exactly fifty years after its first publication.

The standard assignment problem can be seen as a relaxation of more complex combinatorial optimization problems such as traveling salesman problem [5-6], quadratic assignment problem [7], etc. It can also be considered as a particular transportation problem with all supplies and demands equal to 1 . The assignment problem has also several variations such as the semi-assignment problem and the $\mathrm{k}$ cardinality assignment problem. The reader interested in more details about these two problems or other variations can see [3] for a comprehensive survey of the assignment problem variations.

Many optimization problems in real-world applications are multiobjective in nature; indeed a single objective is rarely sufficient to embrace all facets of the problem which should be considered for the evaluation and comparison of 
the alternatives. In that, the assignment problems are not an exception; they can also involve multiple objectives. Indeed, in the problem of assigning jobs to machines, the minimization of completion time can also be an important objective in addition to the conventional objective which is the minimization of assignment cost.

It is worth to indicate that throughout this paper, we indifferently use the terms "multiobjective" and "multicriteria".

In multiobjective optimization problems, there often exist conflicts (contradictions) between the different objectives to be optimized simultaneously. Two objective functions are said to be in conflict if the full satisfaction of one, results in only partial satisfaction of the other. In other words, the optimal solutions for the two objective functions are not the same. There rarely exists a solution that is optimal with respect to all objective functions. If such a particular case arises then only solutions that are optimal with respect to each objective function are efficient. That is why multiobjective optimization problems are said to be ill-defined mathematically. Indeed, an optimal solution i.e. a solution that is optimal with respect to all objective functions rarely exists.

An important issue in multiobjective optimization problems is the determination of efficient (also called Paretooptimal, non-inferior, non-dominated) solutions among which the final solution is to be found. Dominated solutions are excluded from further investigations in the sense that the final solution of the multiobjective optimization problem is looked for among only the efficient solutions.

The final solution of a multiobjective optimization problem depends on the trade-offs between the different conflicting criteria which the decision maker is willing to accept. To calculate what can be considered as the "optimal" solution in such models, additional information is needed - this information can only be obtained from subjective preferences of the decision maker, and the preferred solution is then considered as the "best compromise" solution. The compromise relates to the trade-offs made between the different criteria.

The first paper addressing the multiobjective nature of the assignment problem was published by Charnes et al. in 1969 [8]. Notwithstanding the fact that no solution technique was proposed in this paper, it has however emphasized the importance of considering multiple criteria in assignment problems which has the merit of initiating a new research direction in assignment problems.

Very few publications addressed the problem of determining the efficient solutions of a general multiobjective assignment problem with any number of objective functions and only particular cases such as the biobjective assignment problem [9] were considered. The proposed techniques cannot be easily generalized to the general case of multiobjective assignment problem.

Pentico [3] reported multiobjective assignment problems where the solution techniques either combine all objective functions into a single objective function as in Scarelli and Narula [10] or consider the objective functions separately and sequentially as in Lee and Schniederjans [11]. Both ap- proaches are not suitable to tackle the case where all objective functions are considered simultaneously.

As for other multiobjective optimization problems, an important research issue in multiobjective assignment problems is the one related to the study of efficient solutions.

Since in general the set of efficient solutions can be very large, many of them are not worth to be determined. That is why, we follow a different approach in this paper. Given a feasible solution with interesting characteristics, we determine whether or not this solution is efficient and in the case where it is not efficient we provide an efficient solution which dominates it.

The paper is organized as follows. In the second section we provide the description and formulation of the problem of studying the efficiency of feasible solutions of a multiobjective assignment problem as addressed in this paper. The third section is devoted to the presentation of the method followed to answer the questions raised in this paper and which is based on the transformation of the original problem into an assignment problem with side constraints. In the final section, we give some concluding remarks.

\section{PROBLEM DESCRIPTION AND FORMULATION}

Let us consider an assignment problem with s objective functions denoted as MOAP(s) and formulated as follows:

"min" $\mathrm{z}^{\mathrm{k}}(\mathrm{X})=\sum_{\mathrm{i}=1}^{\mathrm{n}} \sum_{\mathrm{j}=1}^{\mathrm{n}} \mathrm{c}_{\mathrm{ij}}^{\mathrm{k}} \mathrm{X}_{\mathrm{ij}}, \mathrm{k}=1, \ldots, \mathrm{s}$

subject to:

$$
\begin{aligned}
& \sum_{j=1}^{n} X_{i j}=1, i=1, \ldots, n \\
& \sum_{i=1}^{n} X_{i j}=1, j=1, \ldots, n \\
& X_{i j} \in\{0,1\}, i, j=1, \ldots, n
\end{aligned}
$$

"min" is used to indicate that there does not necessarily exists a solution having the minimum value with respect to each objective function and undoubtedly the "optimal" solution will depend on the trade-offs to be made between the different objective functions.

Let $\mathrm{X}^{0}$ be a feasible solution of MOAP(s) i.e., $\mathrm{X}^{0}$ satisfies constraints (2), (3) and (4).

In the first step, we are interested in determining whether or not $\mathrm{X}^{0}$ is an efficient solution of MOAP(s) and in the case where $\mathrm{X}^{0}$ is not efficient, we determine in the second step a solution $\mathrm{X}^{*}$ that is an efficient solution of MOAP(s) and which dominates $X^{0}$. The idea is that the efficiency of $X^{0}$ is investigated because it is assumed to be an interesting solution for the decision maker and its efficiency is checked before its selection as a final solution. In the case where it is not efficient, $\mathrm{X}^{*}$ is not only efficient but it also dominates $\mathrm{X}^{0}$ which makes of it even a better candidate for selection.

Let us recall that $\mathrm{X}^{0}$ is an efficient solution of MOAP(s) if and only if there does not exist any solution $X$ satisfying constraints (2), (3) and (4) such that: (i) $Z^{k}(X) \leq Z^{k}\left(X^{0}\right)$, for 
all $\mathrm{k}=1, \ldots, \mathrm{s}$, and (ii) there is at least one index $\mathrm{h}, 1 \leq \mathrm{h} \leq \mathrm{s}$, for which $Z^{\mathrm{h}}(\mathrm{X})<\mathrm{Z}^{\mathrm{h}}\left(\mathrm{X}^{0}\right)$.

It is worth to notice that $Z^{k}(X) \leq Z^{k}\left(X^{0}\right)$, for all $k=1, \ldots$, $\mathrm{s}$ with at least one strict inequality is equivalent to $Z^{\mathrm{k}}(X) \leq$ $\mathrm{Z}^{\mathrm{k}}\left(\mathrm{X}^{0}\right)$, for all $\mathrm{k}=1, \ldots, \mathrm{s}$ and $\sum_{k=1}^{s} Z^{k}(X)<\sum_{k=1}^{s} Z^{k}\left(X^{0}\right)$.

Consequently, the feasible solution $\mathrm{X}^{0}$ is efficient if the system (S) defined as follows is not feasible.

$\sum_{\mathrm{j}=1}^{\mathrm{n}} \mathrm{X}_{\mathrm{ij}}=1, \mathrm{i}=1, \ldots, \mathrm{n}$

$\sum_{\mathrm{i}=1}^{\mathrm{n}} \mathrm{X}_{\mathrm{ij}}=1, \mathrm{j}=1, \ldots, \mathrm{n}$

$X_{i j} \in\{0,1\}, i, j=1, \ldots, n$

$Z^{k}(X) \leq Z^{k}\left(X^{0}\right), \mathrm{k}=1, \ldots, \mathrm{s}$

$\sum_{k=1}^{s} Z^{k}(X)<\sum_{k=1}^{s} Z^{k}\left(X^{0}\right)$

Let us define $C_{i j}, A_{i j k}$ and $B_{k}$, for $i, j=1, \ldots ., n$, and $k=1$, $\ldots, \mathrm{s}$ as follows:

$\mathrm{C}_{\mathrm{ij}}=\sum_{\mathrm{k}=1}^{\mathrm{s}} \mathrm{c}_{\mathrm{ij}}^{\mathrm{k}}, \mathrm{i}, \mathrm{j}=1, \ldots, \mathrm{n}$

$A_{i j k}=c_{i j}^{k}, i, j=1, \ldots, n ; k=1, \ldots, s$

$\mathrm{B}_{\mathrm{k}}=\mathrm{Z}^{\mathrm{k}}\left(\mathrm{X}_{0}\right), \mathrm{k}=1, \ldots, \mathrm{s}$

Let us define $\mathrm{Z}(\mathrm{X})$ as follows:

$$
\begin{aligned}
Z(X) & =\sum_{k=1}^{s} Z^{k}(X) \\
& =\sum_{k=1}^{s} \sum_{i=1}^{n} \sum_{j=1}^{n} c_{i j}^{k} X_{i j} \\
& =\sum_{i=1}^{n} \sum_{j=1}^{n} \sum_{k=1}^{s} c_{i j}^{k} X_{i j} \\
& =\sum_{i=1}^{n} \sum_{j=1}^{n}\left(\sum_{k=1}^{s} c_{i j}^{k}\right) X_{i j} \\
& =\sum_{i=1}^{n} \sum_{j=1}^{n} C_{i j} X_{i j}
\end{aligned}
$$

With these transformations, we obtain an assignment problem with $\mathrm{s}$ side constraints which we denote as $\operatorname{APSC}\left(\mathrm{X}^{0}\right)$. Its formulation is as follows:

$$
\min Z(X)=\sum_{\mathrm{i}=1}^{\mathrm{n}} \sum_{\mathrm{j}=1}^{\mathrm{n}} \mathrm{C}_{\mathrm{ij}} \mathrm{X}_{\mathrm{ij}}
$$

subject to:

$$
\begin{aligned}
& \sum_{j=1}^{n} X_{i j}=1, i=1, \ldots, n \\
& \sum_{i=1}^{n} X_{i j}=1, j=1, \ldots, n
\end{aligned}
$$

$\sum_{\mathrm{i}=1}^{\mathrm{n}} \sum_{\mathrm{j}=1}^{\mathrm{n}} \mathrm{A}_{\mathrm{ijk}} \mathrm{X}_{\mathrm{ij}} \leq \mathrm{B}_{\mathrm{k}}, \mathrm{k}=1, \ldots, \mathrm{s}$

$X_{i j} \in\{0,1\}, i, j=1, \ldots, n$

It is worth to notice that the number of side constraints in $\operatorname{APSC}\left(\mathrm{X}^{0}\right)$ is equal to the number of objective functions in $\operatorname{MOAP}(\mathrm{s})$.

For the kth side constraint, $\mathrm{k}=1, \ldots, \mathrm{s}$, the left member is simply the kth objective function in $\operatorname{MOAP}(s)$ and the right member is the value of kth objective function for the feasible solution $\mathrm{X}^{0}$.

The objective function of $\operatorname{APSC}\left(\mathrm{X}^{0}\right)$ is the sum of the $\mathrm{s}$ objective functions $\mathrm{Z}^{\mathrm{k}}(\mathrm{X}), \mathrm{k}=1, \ldots, \mathrm{s}$ of the problem $\operatorname{MOAP}(\mathrm{s})$.

$\operatorname{APSC}\left(\mathrm{X}^{0}\right)$ is exactly similar to the $0-1$ assignment problem with side constraints studied by Mazzola and Neebe in [12] and for which they proposed both a branch-and-bound algorithm to solve it to optimality and a heuristic procedure for obtaining approximate solutions.

The branch-and-bound algorithm developed in [12] combines a depth-first polychotomous branching strategy with a bounding procedure utilizing the subgradient optimization.

In the next section, we will see how solving the problem $\operatorname{APSC}\left(\mathrm{X}^{0}\right)$ to optimality can: (i) provide an answer to whether or not the feasible solution of $\operatorname{MOAP}(s) X^{0}$ is efficient and (ii) in the case where it is not efficient determine an efficient solution of MOAP(s) which dominates $\mathrm{X}^{0}$.

\section{PROBLEM SOLVING}

Let $\mathrm{X}^{0}$ be a given feasible solution of $\operatorname{MOAP}(\mathrm{s})$ i.e. $\mathrm{X}^{0}$ satisfies constraints (2), (3) and (4).

It is worth to notice that the set of feasible solutions of MOAP(s) and the set of feasible solutions of SAP are the same. Consequently, the set of feasible solutions of MOAP(s) can be obtained through the use of any solution technique of SAP.

In this section, we address two problems: (i) we investigate whether or not $\mathrm{X}^{0}$ is an efficient solution of $\operatorname{MOAP}(\mathrm{s})$ and (ii) in the case where $\mathrm{X}^{0}$ is found to be not efficient we determine an efficient solution that dominates $\mathrm{X}^{0}$. With respect to each objective function of $\operatorname{MOAP}(s)$, the obtained solution is at least as good as $\mathrm{X}^{0}$.

Let $\mathrm{X}^{*}$ be an optimal solution of $\operatorname{APSC}\left(\mathrm{X}^{0}\right)$ obtained e.g., through the use of the branch-and-bound algorithm of Mazzola and Neebe [12].

Depending on the value of the objective function of $\operatorname{APSC}\left(\mathrm{X}^{0}\right)$ for the optimal solution $\mathrm{X}^{*}$, two cases can be distinguished:

$$
\begin{aligned}
& \sum_{\mathrm{i}=1}^{\mathrm{n}} \sum_{\mathrm{j}=1}^{\mathrm{n}} \mathrm{C}_{\mathrm{ij}} \mathrm{X}_{\mathrm{ij}}^{*}=\sum_{\mathrm{i}=1}^{\mathrm{n}} \sum_{\mathrm{j}=1}^{\mathrm{n}} \mathrm{C}_{\mathrm{ij}} \mathrm{X}_{\mathrm{ij}}^{0}=\sum_{\mathrm{k}=1}^{\mathrm{s}} \mathrm{Z}^{\mathrm{k}}\left(\mathrm{X}^{0}\right) \\
& \sum_{\mathrm{i}=1}^{\mathrm{n}} \sum_{\mathrm{j}=1}^{\mathrm{n}} \mathrm{C}_{\mathrm{ij}} \mathrm{X}_{\mathrm{ij}}^{*} \prec \sum_{\mathrm{i}=1}^{\mathrm{n}} \sum_{\mathrm{j}=1}^{\mathrm{n}} \mathrm{C}_{\mathrm{ij}} \mathrm{X}_{\mathrm{ij}}^{0}=\sum_{\mathrm{k}=1}^{\mathrm{s}} \mathrm{Z}^{\mathrm{k}}\left(\mathrm{X}^{0}\right)
\end{aligned}
$$

In the first case (12), the feasible solution $\mathrm{X}^{0}$ is efficient because any feasible solution of $\operatorname{MOAP}(\mathrm{s})$ which is at least 
as good as $\mathrm{X}^{0}$ with respect to all criteria i.e., fulfils the constraints of $\operatorname{APSC}\left(\mathrm{X}^{0}\right)$ cannot be strictly better than $\mathrm{X}^{0}$ on one of the s criteria. Indeed, if there exist a solution $X^{\prime}$ that is feasible for $\operatorname{APSC}\left(\mathrm{X}^{0}\right)$ and an index $\mathrm{h}, 1 \leq \mathrm{h} \leq \mathrm{s}$, such that $\mathrm{Z}^{\mathrm{h}}\left(\mathrm{X}^{\prime}\right)<$ $\mathrm{Z}^{\mathrm{h}}\left(\mathrm{X}^{0}\right)$, then:

$$
\sum_{\mathrm{i}=1}^{\mathrm{n}} \sum_{\mathrm{j}=1}^{\mathrm{n}} \mathrm{C}_{\mathrm{ij}} \mathrm{X}_{\mathrm{ij}}^{\mathrm{\prime}} \prec \sum_{\mathrm{k}=1}^{\mathrm{s}} \mathrm{Z}^{\mathrm{k}}\left(\mathrm{X}^{0}\right)=\sum_{\mathrm{k}=1}^{\mathrm{s}} \mathrm{Z}^{\mathrm{k}}\left(\mathrm{X}^{*}\right)
$$

contradicts the fact that $\mathrm{X}^{*}$ is an optimal solution to $\operatorname{APSC}\left(\mathrm{X}^{0}\right)$.

In the second case (13), the feasible solution $X^{0}$ is not efficient and $X^{*}$ is an efficient solution that dominates $X^{0}$. Indeed, since $Z^{k}\left(X^{*}\right) \leq Z^{k}\left(X^{0}\right)$, for all $k=1, \ldots$, s because $X^{*}$ satisfies constraints (11), and (13) is fulfilled then there necessarily exists an index $h, 1 \leq h \leq s$, such that $Z^{h}\left(X^{*}\right)<Z^{h}\left(X^{0}\right)$. Consequently, $\mathrm{X}^{*}$ dominates $\mathrm{X}^{0} . \mathrm{X}^{*}$ is an efficient solution for $\operatorname{MOAP}(s)$. Indeed, if $X^{*}$ is not efficient, then there will exist $X$ satisfying (2), (3), and (4) such that $Z^{k}(X) \leq Z^{k}\left(X^{*}\right)$, for all $k=$ $1, \ldots, \mathrm{s}$ and

$$
\sum_{\mathrm{k}=1}^{\mathrm{s}} \mathrm{Z}^{\mathrm{k}}(\mathrm{X}) \prec \sum_{\mathrm{k}=1}^{\mathrm{s}} \mathrm{Z}^{\mathrm{k}}\left(\mathrm{X}^{*}\right)
$$

Since for all $\mathrm{k}=1, \ldots, \mathrm{s}, \mathrm{Z}^{\mathrm{k}}(\mathrm{X}) \leq \mathrm{Z}^{\mathrm{k}}\left(\mathrm{X}^{*}\right)$ and $\mathrm{Z}^{\mathrm{k}}\left(\mathrm{X}^{*}\right) \leq$ $Z^{k}\left(X^{0}\right)$, then necessarily $Z^{k}(X) \leq Z^{k}\left(X^{0}\right)$ for all $k=1, \ldots$, s. Hence, $X$ satisfies (11). Since $X$ satisfies also (2), (3), and (4), then $X$ is a feasible solution of APSC $\left(X^{0}\right)$. The fact that $X^{*}$ is an optimal solution of APSC $\left(\mathrm{X}^{0}\right)$ implies that $\sum_{\mathrm{k}=1}^{\mathrm{s}} \mathrm{Z}^{\mathrm{k}}\left(\mathrm{X}^{*}\right) \leq \sum_{\mathrm{k}=1}^{\mathrm{s}} \mathrm{Z}^{\mathrm{k}}(\mathrm{X})$ which contradicts condition $(15)$.

We have shown that solving APSC $\left(\mathrm{X}^{0}\right)$ to optimality is a sufficient means to answer the question "Is $\mathrm{X}^{0}$ an efficient solution?" and to determine an efficient solution that dominates $\mathrm{X}^{0}$ in the case where $\mathrm{X}^{0}$ is not efficient.

\section{CONCLUSIONS}

Real-life assignment problems may involve more than a single objective function to optimize that is why multiobjective assignment models suit better to real-life assignment applications than single objective assignment models. An important concept in multiobjective optimization is the one related to efficient solutions.

In this paper, we investigated the problem of knowing whether or not a given feasible solution of a multiobjective assignment problem is efficient and in the case where it is not efficient we provided a technique to determine an efficient solution that dominates it. A fortiori, the later is at least as good as the former since it has equal or better performances on all criteria.

The way in which the problem of efficiency of feasible solutions of a multiobjective assignment problem is tackled in this paper is different from the conventional one where all efficient solutions are determined in a first step and the final solu- tion is looked for among them in a second step. It should be noticed that until now there does not exists any technique that can determine all efficient solutions of a general multiobjective assignment problem; only particular cases such as the biobjective assignment problem are dealt with in the literature.

We provided a means for answering the two questions raised in this paper through the transformation of the original problem into an assignment problem with side constraints similar to the one considered in [12] for which there exists a branch-and-bound algorithm that solve it to optimality. It is the comparison between the feasible solution of the multiobjective assignment problem and the optimal solution(s) of the induced assignment problem with side constraints that allow to answer the two questions.

The main application of the work developed in this paper is mainly the case where an interesting feasible solution of a multiobjective assignment problem is given and where its selection as a final solution is conditioned by the fact whether or not it is an efficient solution.

It is important to notice that this work provides an additional potential application of the solution techniques of the assignment problem with side constraints and particularly those solving it to optimality.

\section{ACKNOWLEDGEMENTS}

This work is part of a dissertation for obtaining Licence Spéciale in Mathematics Applied to Management from the Free University of Brussels. I would like to thank Professor Philippe Vincke who supervised this dissertation.

\section{REFERENCES}

[1] Sonia, Puri MC. Two-stage time minimizing assignment problem. Omega 2008; 36(5): 730-740

[2] Kuhn HW. The Hungarian method for the assignment problem. Nav Res Logist Quart 1955; 2(1-2): 83-97.

[3] Pentico DW. Assignment problems: A golden anniversary survey. Eur J Opl Res 2007; 176(2): 774-793.

[4] Kuhn HW. The Hungarian method for the assignment problem. Nav Res Logist Quart 2005; 52(1): 7-21.

[5] Eastman WL. A solution to the traveling salesman. Econometrica 1959; 27: 28-292.

[6] Balas E, Christofides N. A restricted lagrangean approach to the traveling salesman problem. Math Prog 1981;21: 19-46.

[7] Bazaraa MS, Elshafei AN. An exact branch-and-bound procedure for the quadratic assignment problem. Nav Res Logist Quart 1979; 26 109-121.

[8] Charnes A, Cooper WW, Neihaus RJ, Stedry A. Static and dynamic assignment models with multiples objectives and some remarks on organization design. Manag Sci 1969; 15(8): 365-375.

[9] Malhotra R, Bhatia ML, Puri MC. Bi-criteria assignment problem. Opsearch 1982; 19(2): 84-96.

[10] Scarelli A, Narula SC. A multicriteria assignment problem. J Multi Crit Decis Anal 2002; 11(2): 65-74.

[11] Lee SM, Schniederjans MJ. A multicriteria assignment problem: A goal programming approach. Interfaces 1983; 13(4): 75-81.

[12] Mazzola JB, Neebe AW. Resource-constrained assignment scheduling. Oper Res 1986; 34(4): 560-572. 